\title{
Análise da percepção dos gestores e dos profissionais envolvidos no Projeto Academia da Saúde sobre os processos de implantação, acompanhamento e avaliação
}

\author{
Perception analysis of the managers and professionals involved in the Health Academy \\ Project on the implementation, follow-up and evaluation processes
}

\begin{abstract}
Análisis de la percepción de los gestores y de los profesionales del Proyecto Academia de Salud sobre los procesos de implantación, acompañamiento y evaluación
\end{abstract}

Tiago Aparecido de Souza Lopes ${ }^{1 *}$, Saulo Daniel Mendes Cunha².

\section{RESUMO}

Objetivo: O presente estudo tem o objetivo de analisar como os gestores e profissionais envolvidos no Projeto, Academia da Saúde percebem o desenvolver dessa estratégia, que visa à promoção da saúde de forma integral. Métodos: A presente pesquisa foi desenvolvida por meio da abordagem qualitativa. Participaram cinco profissionais, sendo: um coordenador, um secretário, um professor e dois estagiários envolvidos no programa. A coleta dos dados foi realizada nos meses de agosto e de setembro de 2018 e, para a entrevista, foi utilizado um roteiro semi-estruturado e para o registro das entrevistas, foi utilizado um gravador da marca Coby Cvr20 a fim de gravar, para posterior análise, todas as informações fornecidas pelos participantes. Para análise dos dados, as falas foram agrupadas em categorias e, posteriormente, os dados foram analisados por meio da técnica de análise do discurso. Resultados: Os envolvidos no estudo percebem a importância dessa estratégia e apontam obstáculos que dificultam sua execução, como a falta de profissionais suficientes para atender à demanda da população, o que prejudica diretamente 0 desenvolvimento desse projeto, impossibilitando o acompanhamento e a avaliação eficientes. Considerações Finais: Sugerimos que haja melhor articulação entre os níveis de gestão a fim de driblar os entraves que dificultam o desenvolver dessa estratégia, através do apoio à implementação de ações que possam fortalecer o programa e uma melhor gestão de recursos humanos e financeiros.

Palavras Chave: Políticas Públicas, Saúde, Gestão, Educação Física.

\begin{abstract}
Objective: This study aims to analyze how the managers and professionals involved in the Health Academy Project perceive the development of this strategy, which aims at the promotion of health in a comprehensive way. Methods: The present research was developed through the qualitative approach. Five professionals participated, being: a coordinator, a secretary, a teacher and two trainees involved in the program. Data collection was performed in August and September 2018, and for the interview, a semi-structured script was used and for recording interviews, a Coby Cvr20 recorder was used in order to record, for later analysis, all the information provided by participants. For the analysis of the data, the statements were grouped into categories and, later, the data were analyzed through the discourse analysis technique. Results: Those involved in the study perceive the importance of this strategy and point out obstacles that impede its execution, such as the lack of professionals sufficient to meet the population demand, which directly hinders the
\end{abstract}

\footnotetext{
${ }^{1}$ Graduando em Educação Física pela Universidade Estadual de Montes Claros - UNIMONTES. Montes Claros - MG.

2 Docente da Universidade Estadual de Montes Claros - UNIMONTES. Montes Claros - MG.

*E-mail: thiagocido@hotmail.com
} 
development of this project, making efficient monitoring and evaluation impossible. Conclusion: We suggest a better articulation between levels of management in order to overcome the obstacles that hamper the development of this strategy, by supporting the implementation of actions that can strengthen the program and better management of human and financial resources.

Keywords: Public Policies, Health, Management, Physical Education.

\section{RESUMEN}

Objetivo: Este estudio tiene por objetivo analizar cómo los gestores y los profesionales involucrados en el Proyecto, Academia de Salud perciben el desarrollo de dicho proyecto, que persigue la promoción de la salud de una forma integral. Métodos: Esta pesquisa fue desarrollada con un abordaje cualitativo. Participaron de la misma cinco profesionales: un coordinador, un secretario, un profesor y dos pasantes participantes en dicho programa. La recolección de los datos se realizó entre agosto y septiembre del 2018. para la entrevista, fue utilizado un plan semiestructurado y para el registro de las entrevistas, una grabadora Coby Cvr20 a fin de grabar, para su posterior análisis, todas las informaciones ofrecidas por los encuestados. Para el análisis de los datos, los testimonios fueron agrupados en categorías y, posteriormente, analizados por medio del análisis del discurso. Resultados: Los participantes en el estudio perciben la importancia de ese proyecto y apuntan los obstáculos que dificultan su ejecución, tales como la falta de profesionales suficientes para atender la demanda de la población lo que perjudica directamente su desarrollo imposibilitando el acompañamiento y una evaluación eficientes. Conclusión: Sugerimos una mejor articulación entre los niveles de gestión a fin de evadir los obstáculos que dificultan el desarrollo del proyecto, por medio del apoyo a la implementación de acciones que fortalezcan dicho programa así como una administración mejor de los recursos humanos y financieros.

Palabras Clave: Políticas Públicas, Salud, Gestión, Educación Física.

\section{INTRODUÇÃO}

As políticas públicas correspondem ao desenvolvimento de ações que objetivam sanar problemas e necessidades de saúde de uma população. Trata-se de um conjunto de estratégias desenvolvidas pelo Estado que visam à garantia de direitos assegurados constitucionalmente. O direito à saúde está elencado na Constituição Federal em seu artigo 196, sendo dever do Estado a garantia desse direito mediante políticas que visem à redução do risco de doenças e agravos (BRASIL, 2010; BRASIL, 1988).

Nesse sentido, as políticas públicas de saúde são intervenções criadas tendo como objetivo principal a promoção da saúde da população por meio de parcerias com órgãos federais, estaduais, municipais e também com a sociedade a fim de favorecer o desenvolvimento integral das ações de promoção da saúde (SALDANHA et al., 2016).

A política de promoção da saúde se insere no âmbito da Saúde Pública como uma ferramenta para auxiliar na prevenção de doenças, principalmente das doenças crônicas degenerativas as quais podem ser amenizadas através de hábitos de vida saudáveis, melhorando, assim, a saúde da população em geral (KOWALSKI, 2013).

A saúde e a atividade física estão intimamente ligadas, sendo esta relação fundamental para a melhoria da qualidade de vida e, muitas vezes, por uma diversidade de fatores, sendo eles socioeconômicos ou, até mesmo, por falta de tempo, boa parte da população não tem acesso a uma prática de atividade física com orientação. Diante dessa realidade, surgiram estratégias para que a promoção da saúde ocorra de forma integral e contínua (WEBER et al., 2017).

Nessa perspectiva, foi implantado numa cidade do Norte de Minas, em agosto de 2016. O Projeto Academia da Saúde se insere na ideia de tornar mais acessível à população um meio de prática regular de atividade física. Essa estratégia tem como objetivo principal a contribuição da promoção da saúde e do cuidado, através do incentivo de práticas de vida saudáveis por profissionais qualificados, que atuam em estruturas adequadas e construídas especialmente para esse fim denominadas polos (BRASIL, 2014).

REAS/EJCH | Vol. 11 (8) | e543 | DOI: https://doi.org/10.25248/reas.e543.2019 Página 2 de 8 
O presente estudo justificou-se pela necessidade de analisar como os gestores e os profissionais envolvidos no Projeto Academia da Saúde percebem o desenvolver dessa estratégia que visa à promoção da saúde de forma integral, já que trata-se de um importante política pública implantada recentemente, verificando-se a relevância de se conhecer o desenvolvimento dessa estratégia. Dessa forma, este estudo tem como objetivo analisar a percepção dos gestores e dos profissionais envolvidos no projeto Academia da Saúde sobre os processos de implantação, acompanhamento e avaliação.

\section{MÉTODOS}

A presente pesquisa foi desenvolvida por meio da abordagem qualitativa que para Minayo et al., (2008) é a modalidade de estudo se preocupa com um nível de realidade que não pode ser quantificado. Os sujeitos do estudo foram os profissionais que atuam no projeto da cidade onde o estudo foi realizado.

Participaram cinco profissionais, sendo: um coordenador, um secretário, um professor e dois estagiários envolvidos no programa e que atuam nas zonas urbana e rural da cidade. Para inclusão na pesquisa, o participante deveria estar envolvido diretamente com o projeto e assinar o Termo de Consentimento Livre e Esclarecido.

A coleta dos dados foi realizada nos meses de agosto e de setembro de 2018 e, buscando atingir o objetivo proposto, foi utilizada como instrumento de coleta de dados a entrevista individual com roteiro semiestruturado. Para o registro das entrevistas, foi utilizado um gravador da marca Coby Cvr20 a fim de gravar, para posterior análise, todas as informações fornecidas pelos participantes. Além da confiabilidade, esse método possibilita, ao escutar novamente o conteúdo gravado, aperfeiçoamento e destaque das ideias expostas pelo informante (TRIVIÑOS, 1994)

Para a análise dos dados, o conteúdo coletado na entrevista semi-estruturada foi transcrito na íntegra e, após transcrição e leitura dos relatos, foi utilizado o método da categorização dos dados proposta por Minayo (2008, p.70) que diz: "trabalhar com categorias significa agrupar elementos, ideias ou expressões em torno de um conceito capaz de abranger tudo isso". Os dados foram analisados por meio da técnica de análise do discurso.

A fim de se garantir o anonimato dos sujeitos, serão utilizados números de identificação, os quais serão identificados como (G1, G2, P3, P4 e P5) sendo G de gestores e P de profissionais e o número de acordo com a ordem em que foram entrevistados.

Por se tratar de uma pesquisa envolvendo seres humanos, o projeto foi submetido a um Conselho de Ética em Pesquisa que foi aprovado mediante Parecer Consubstanciado ํㅜ 2.536.188. O estudo respeitou os princípios e as exigências formais contidas em normas que regulam as pesquisas envolvendo seres humanos. O aceite da participação se deu através da assinatura do Termo de Consentimento Livre e Esclarecido.

\section{RESULTADOS E DISCUSSÃO}

O Programa Academia da Saúde no município onde o estudo foi realizado é vinculado à atenção primária, como preconiza o Ministério da Saúde, e conta com onze polos do projeto Academia da Saúde. As ideias consideradas mais relevantes foram selecionadas e grupadas em quatro categorias, as quais foram: a implantação do Projeto Academia da Saúde, avaliação e acompanhamento dos participantes, obstáculos encontrados para execução do Projeto e, por fim a implantação do projeto e a melhoria da qualidade de vida dos seus participantes.

\section{A Implantação Do Projeto Academia Da Saúde}

A forma de disponibilizar os serviços de saúde e a maneira como eles organizam vêm sofrendo mudanças devido à mudança do perfil da saúde. Num cenário em que o adoecimento e a mortalidade por condições crônicas vêm prevalecendo, faz-se necessária a criação de estratégias para reverter essa situação. Desde os anos 2000 , estão sendo criados programas de incentivo à prática de atividade física em locais públicos como

REAS/EJCH | Vol. 11 (8) | e543 | DOI: https://doi.org/10.25248/reas.e543.2019 Página 3 de 8 
uma forma de enfrentamento do sobrepeso e da obesidade. Essas iniciativas embasaram a criação do Programa Academia da Saúde no ano de 2011 (BRASIL, 2014).

A implantação do Programa Academia da Saúde constitui uma das formas de efetivação da Política Nacional de Promoção da Saúde no Brasil. Esse programa atua através da implementação de polos com infraestrutura, equipamentos e quadro de pessoal capacitado para a orientação da prática de atividade física, lazer e hábitos de vida saudáveis, possibilitando uma popularização de equipamentos de ginástica nos espaços públicos (BRASIL, 2014).

Sobre a implantação desse programa na cidade estudada, os gestores relataram que o projeto trata-se de uma política pública criada pelo governo com adesão pelo município.

“...esse projeto é uma estratégia criada pelo governo com o objetivo de tornar a prática de atividade física mais acessível à população local..." (G2).

“...é um projeto do governo que vem sendo ampliado cada dia mais para melhorar a qualidade de vida das pessoas." (P5).

Os profissionais envolvidos no projeto compreendem a importância da implantação dessa estratégia para melhoria da saúde da população de uma maneira geral, uma vez que, além de contribuir para a prevenção e o controle de doenças crônico-degenerativas, a prática regular de atividade física contribui para o bem estar físico, mental e social do indivíduo. Essa ideia está intimamente ligada ao conceito de saúde, estabelecido pela Organização Mundial de Saúde - OMS que a define como "um completo estado de bem estar físico, mental e social e não somente a ausência de doenças" (OMS, 1946).

Nesse sentido, com objetivo principal de se prevenirem as doenças crônico-degenerativas, surge essa política de promoção da saúde, fazendo parte da Saúde Pública como uma ferramenta para a melhoria da saúde população em geral por meio do incentivo de hábitos de vida saudáveis (KOWALSKI, 2016).

De acesso livre à população, o projeto se configura como um ponto da Rede de Atenção. Nesse sentido, o desenvolvimento de suas ações complementa o cuidado individual e coletivo (BRASIL, 2014).

Através da análise das falas abaixo, parece que já existia na cidade um projeto de prática de atividade física ao ar livre e posteriormente houve uma adesão oficial a essa política pública do Ministério da Saúde. Veja o relato abaixo:

“...é um programa primário do governo, tivemos a iniciativa, a ideia de colocar profissionais da área de Educação Física para desenvolver o trabalho de alongamentos, aeróbica, melhorando a qualidade de vida, da Saúde, prevenindo e fazendo atenção primária de prevenção à saúde para idosos, crianças, adultos, todo público em geral..." (G1).

O principal objetivo dessa política pública é o estímulo de hábitos de vida saudáveis e a qualidade de vida. Dessa forma a formulação e a reformulação de estratégias se fazem necessárias no âmbito da saúde e devem ir além do olhar voltado apenas para as doenças (COSTA et al., 2013).

Sob esse aspecto, é importante ressaltar que essa etapa de implementação deve ser entendida como uma etapa essencial dentro da análise de uma política pública visto que ela representa a oportunidade de entender os processos implicados para alcançar os efeitos esperados na etapa de elaboração da estratégia (DALFIOR et al.,2015).

\section{Avaliação E Acompanhamento Dos Participantes Do Projeto Academia Da Saúde}

Uma avaliação é fundamental para verificar o processo como um todo, uma vez que, através dela, é possível verificar tanto, se o serviço ou intervenção estão sendo ofertados adequadamente, como também se os participantes estão efetivamente fazendo uso do serviço (OLIVEIRA et al., 2014).

No que se refere às avaliações e ao acompanhamento dos participantes do projeto, os profissionais envolvidos afirmaram que existe uma avaliação dos participantes ao aderir ao programa.

REAS/EJCH | Vol. 11 (8) | e543 | DOI: https://doi.org/10.25248/reas.e543.2019 Página 4 de 8 
“...existe avaliação de diversos profissionais como Nutricionista e Educador Físico, quando uma pessoa entra é feito um acompanhamento primário do participante, para ver se ele tem um problema de saúde..." (G2).

"...se a pessoa tiver interesse para entrar e houver vagas, ela vai passar por uma avaliação primária e, depois de avaliado, se não tiver nenhum problema, ele já pode participar mas caso ele tenha algum problema, ele é orientado a procurar o Programa de Saúde da Família e, depois que ele esteja apto a praticar atividade física, já pode ser incluso em nosso programa." (G3).

Dessa forma, parece haver o entendimento por, parte dos envolvidos no estudo, sobre importância desse projeto como também a importância do acompanhamento e uma avaliação dos participantes. No entanto, não existe uma padronização no que diz respeito à frequência ou à periodicidade dessas avaliações. Nesse sentido, parece que há obrigatoriedade apenas quando o indivíduo é inserido no programa. Para Oliveira et al., 2014 a avaliação primária é fundamental para verificar a possibilidade de ocorrência de eventos adversos relacionados ao exercício e também elaborar recomendações específicas acerca do início, continuação ou da progressão de um programa de atividade física

No programa estudado, existe a avaliação primária do participante ao entrar no projeto, o que é essencial, a fim de se evitarem complicações, principalmente em pacientes que sofrem de problemas crônicos, como hipertensão entre outras doenças cardiovasculares. Ademais, existe também a necessidade do acompanhamento frequente dos participantes para avaliação dos resultados, bem como para avaliar a evolução do estado de saúde. O grupo de gestores e de profissionais envolvidos colocam que:

“... Onde eu estou, que é na zona rural, nos polos de lá eu ainda não vi o acompanhamento, porém não tem muito tempo que estou nesse projeto... eles passam pelo processo de pesar, medir, passa com a Nutricionista (P5).

“...é feito um acompanhamento com Nutricionista e o Educador Físico o acompanhamento aqui na zona urbana ocorre com uma frequência maior que na zona rural, mas lá também tem o acompanhamento normalmente." (G2).

“..., são reavaliados semanalmente” (P3).

"...é uma vez por acaso, duas vezes no mês mas não temos fichas de controle destes pacientes." (P4).

Diante disso, esses profissionais evidenciam a existência de reavaliações durante a participação no projeto, porém, chama atenção quanto à falta de periodicidade e de uma padronização quanto ao que é avaliado e quais profissionais que compõem essas avaliações. Ainda é válido ressaltar que não existe nenhuma documentação oficial, como fichas, para acompanhamento dos participantes.

Silva et al. (2017) afirma que essa falta de documentação específica pode estar relacionada ao fato de se tratar de um programa relativamente novo, cuja implantação ocorreu, de fato, somente em municípios em que foram incorporadas iniciativas de estratégias já existentes anteriormente.

Por se tratar de uma política pública, avaliações periódicas dos participantes são necessárias uma vez que além de verificar a evolução do estado de saúde dos participantes, permite aos gestores envolvidos verificar a execução da intervenção. A avaliação e o acompanhamento em um projeto são essenciais, uma vez que permitem que os profissionais envolvidos com o fazer em saúde se apropriem de ferramentas que possibilitem um diagnóstico acerca da funcionalidade e do prosseguimento das práticas e dos serviços oferecidos, bem como a construção de estratégias de intervenção acerca dos obstáculos identificados (ELIKER; KRUG, 2015).

Uma avaliação eficiente seria fundamental, pois, através dos resultados de evolução de saúde dos participantes seria possível embasar a avaliação como um todo, verificando a funcionalidade do programa no município. Para Dalfior et al. (2015), a avalição consiste em fazer uma análise sobre uma intervenção ou

REAS/EJCH | Vol. 11 (8) | e543 | DOI: https://doi.org/10.25248/reas.e543.2019 Página 5 de 8 
sobre qualquer um dos seus componentes, objetivando auxiliar a tomada de decisões, aumentar o controle sobre a intervenção bem como satisfazer as exigências dos organismos financiadores.

É importante ressaltar que, como prevê o Ministério da Saúde, o Programa Academia da Saúde é um componente da atenção básica, fazendo parte da linha do cuidado e não deve ser tratado como um serviço isolado. Mas para isso, os profissionais da saúde, especialmente os que compõem os Núcleos de Apoio à Saúde da Família - NASF's deverão incluir essa estratégia no planejamento conjunto de suas ações (BRASIL, 2014).

\section{Obstáculos Encontrados Para Execução Do Projeto Academia Da Saúde}

Por ser uma política pública de abrangência nacional, é natural que sejam encontradas dificuldades para a execução do projeto, devido às particularidades que cada local apresenta. Quanto aos obstáculos mostrados no estudo, houve uma resposta unanime entre os envolvidos no estudo que foi a questão territorial relacionada à falta de profissionais como apresentam os discursos seguintes:

“...tem o fato de que por aqui ser um território muito grande não tem a quantidade suficiente de profissionais para atender à quantidade de pessoas que a gente gostaria de atender."(G2).

“...se tivesse mais com certeza seria bem melhor para executar o projeto daria para atender uma área bem maior de pessoas principalmente na área rural." (P5).

Dessa forma, os indivíduos do estudo parecem apresentar como dificuldades: a falta de cobertura territorial da zona urbana e zona rural e a escassez de profissionais para o atendimento neste projeto, uma vez que apresenta número insuficiente de profissionais para atender à demanda da população. Para Falci e Belisário (2013), a fim de que haja qualidade do serviço prestado pelo programa Academia da Saúde é fundamental que haja melhorias principalmente na quantidade de profissionais da área da saúde como professores de Educação Física e Nutricionistas para a devida orientação e acompanhamento dos usuários.

Essa escassez de profissionais constitui um fator que pode acarretar limitações para o bom funcionamento dessa política, uma vez que além de não possibilitar o atendimento de uma maior parte da população pode afetar a qualidade do serviço prestado à população já atendida.

Os discursos demonstram que a área rural é a região que mais se torna prejudicada devido ao déficit de profissionais. O município onde foi realizado o estudo conta com somente cinco profissionais que estão ligados diretamente a essa política, para atender às zonas urbana e rural.

De acordo com o Ministério da Saúde dos profissionais que comporão cada polo vinculado ao Núcleo de Apoio à Saúde da Família, pelo menos 1 (um) profissional de saúde de nível superior, com carga horária de 40 horas semanais ou 2 (dois) profissionais de saúde de nível superior com carga horária mínima de 20 horas semanais cada, que será(ao) responsável(is) pelas atividades do Programa Academia da Saúde (BRASIL, 2013). Dessa forma, a quantidade de profissionais parece estar aquém do esperado, visto que a cidade conta com onze polos do programa. Outra dificuldade apresentada por um dos envolvidos no estudo foi a insuficiência de recursos e de equipamentos que ocasionalmente são utilizados nessa estratégia, como mostram os discursos abaixo:

"Se o governo mandasse mais recursos financeiros o projeto, que eu já considero bom daria para ser executado de uma forma melhor..." (G2).

“...outra coisa que precisa também é que tenha um maior investimento nesse projeto, é necessário, por exemplo, que tenha mais recursos para a compra de outros pequenos equipamentos." (P5).

Os gestores relatam que, além da escassez de profissionais, a falta de recursos financeiros, também apresenta um desafio para o Programa. No processo de implementação de ações de uma política pública, é necessário que haja o envolvimento de diversos atores para que haja uma melhor focalização e destinação de recursos para atender de forma satisfatória à política (SILVA et al., 2017)

REAS/EJCH | Vol. 11 (8) | e543 | DOI: https://doi.org/10.25248/reas.e543.2019 Página 6 de 8 


\section{A Implantação Do Projeto Academia Da Saúde E A Melhoria Da Qualidade De Vida Dos Seus Participantes}

É evidente que a prática regular de exercícios físicos tem importância significativa em todas as fases da vida, trazendo benefícios que contribuem para a melhoria da saúde como um todo, desde o nível físico ao psicológico, resultando na melhoria da qualidade de vida dos indivíduos (SCHEFFER et al., 2015).

Outro entendimento que se pode ter, através dos discursos dos envolvidos no estudo, foi que estes percebem o impacto que essa política pública tem na melhoria da qualidade de vida dos usuários, não somente em âmbito físico e biológico, como também tem uma influência bastante positiva no bem estar psicológico dos participantes. Além disso, de favorece o lazer e a interação social visto que o município conta com poucas atividades para entretenimento.

Ao se encarregarem de promover saúde à população de um determinado território, as equipes envolvidas na esfera assistencial devem se desviar do antigo modelo biomédico tradicional. Devem promover também a qualidade de vida dos indivíduos (FREITAS; MANDU, 2010). É perceptível que a prática de atividade física tem influência tanto na prevenção de doenças crônico-degenerativas quanto na contribuição positiva para o lado psicossocial dos indivíduos como se pode notar nos discursos seguintes

“...as participações no projeto têm uma influência muito positiva no emocional das pessoas porque são criados ciclos de amizades e por ser um local onde não tem muitas opções de lazer, o projeto acaba sendo o lazer para as pessoas daqui." (G2)

“...o projeto, ele é mais que prevenção da Saúde em termos físico, fisiológicos, ele passou a ter prioridade maior na questão do lazer e o entretenimento das pessoas..." (P3)

“...O projeto além de ajudar na melhoria da saúde dos participantes ele funciona como um entretenimento para os participantes especialmente na zona rural por não ter muitos recursos..." (P4).

A utilização de academias de ginástica tem se tornado uma prática cada vez mais difundida entre as pessoas, sendo a forma mais eficaz de promoção de atividade física, que, muitas vezes, por diversos fatores não está presente na rotina diária do ser humano. A junção das práticas de condicionamento físico ao suporte público de lazer permite, além do acesso de toda população, uma união de faixas etárias, ritmos e objetivos diferentes nesses espaços (CASTAÑON et al., 2016).

Em um estudo realizado por Oliveira (2015), comprovou-se que projetos com essa modalidade (em espaços públicos), possuem também um caráter lúdico e estão vinculados aos cuidados em saúde. Além de ser marcado por forte sociabilidade, que pode contribuir para uma experiência coletiva gregária entre as participantes, constitui também um fator motivador para continuarem frequentando o projeto. Dessa forma, os espaços onde ocorrem os programas de prática de atividade física constituem locais muito importantes para a população, uma vez que contribui não somente para a prevenção de doenças crônicas como também melhoram o bem estar e desenvolvem o lazer dos usuários (NOGUEIRA; FERNANDES, 2013).

\section{CONCLUSÃO}

Os envolvidos neste estudo percebem a importância dessa estratégia que, desde que foi implantada no município estudado, vem trazendo inúmeros benefícios aos usuários. Porém, foram apontados, pelos profissionais envolvidos, obstáculos que dificultam uma melhor funcionalidade dessa intervenção, como a escassez de profissionais para atender à demanda o que prejudica, não somente a população desassistida, como também torna difícil um acompanhamento efetivo e avaliações periódicas dos usuários. Situação esta que foi percebida nos depoimentos já que não há uma periodicidade ou padrão para acompanhamentos dos participantes. Diante dos resultados apresentados, é preciso que haja uma melhor articulação entre os níveis de gestão a fim de driblar os entraves que dificultam o desenvolver dessa estratégia, através do apoio à implementação de ações que possam fortalecer o programa e uma melhor gestão de recursos humanos e

REAS/EJCH | Vol. 11 (8) | e543 | DOI: https://doi.org/10.25248/reas.e543.2019 Página 7 de 8 
financeiros. Isso porque, investir em programas de prevenção significa minimizar posteriores custos elevados com a atenção secundária e terciária à saúde.

\section{REFERÊNCIAS}

1. BRASIL. Constituição da República Federativa do Brasil: promulgada em 5 de outubro de 1988. Brasília, DF: Senado Federal, 1988.

2. BRASIL. Ministério da Saúde. Academia da Saúde - Cartilha normativa. Secretaria de Atenção à Saúde do Minstério da Saúde Brasília: 2014

3. BRASIL. Ministério da Saúde. Infraestrutura dos polos. Secretaria de Atenção à Saúde, Departamento de Atenção Básica. Brasília, 2014.

4. BRASIL. Ministério da Saúde. Portaria GM/MS oㅜ 2.684, de 8 de novembro de 2013. Redefine as regras e os critérios referentes aos incentivos financeiros de investimento para construção de polos e de custeio no âmbito do Programa Academia da Saúde e os critérios de similaridade entre Programas em Desenvolvimento no Distrito Federal ou no município e o Programa Academia da Saúde. Brasília, 2013.

5. BRASIL. Ministério da Saúde. Secretaria de Vigilância em Saúde. Secretaria de Atenção à Saúde. Política Nacional de Promoção da Saúde / Ministério da Saúde, Secretaria de Vigilância em Saúde, Secretaria de Atenção à Saúde. - 3. ed. Brasília: Ministério da Saúde, 2010.

6. CASTAÑON JAB, PAIVA CS, FONSECA KM, CARNEIRO RS. Academias ao Ar Livre: uma análise dos espaços públicos. Congresso Nacional de Ergonomia Aplicada. 2016: 1-12.

7. COSTA BVL, MENDONÇA RD, SANTOS LC, PEIXOTO SV, ALVES M, LOPES ACS. Academia da Cidade: um serviço de promoção da saúde na rede assistencial do Sistema Único de Saúde. Ciência e Saúde Coletiva, 2013; 18 (1): 95-102.

8. DALFIOR ET, LIMA RCD, ANDRADE MAC. Reflexões sobre análise de implementação de políticas de saúde. Saúde Debate, 2015; 39 (104): 210-225

9. ELIKER G, KRUG MR. Políticas públicas de promoção da saúde através da atividade física.Um estudo de caso em dois municípios do Coredi Alto Jacuí. Revista Interdisciplinar de Ensino Pesquisa e Extensão, 2015; 6(2):1-12.

10. FALCI DM, BELISÁRIO SA. A inserção do profissional de educação física na atenção primária à saúde e os desafios em sua formação. Interface, 2013; 17 (47): 885-899.

11. FREITAS MLA, MANDÚ ENT, Promoção da saúde na Estratégia Saúde da Família: análise de políticas de saúde brasileiras. Acta Paulista de Enfermagem, 2010; 23(2): 200-205.

12. KOWALSKI AIC. Políticas de promoção da saúde, atividade física e envelhecimento humano no município de Derrubadas RS. Trabalho de Conclusão de Curso. Universidade Regional do Noroeste do Estado do Rio Grande do Sul, ljuí, 2016; 46p.

13. MINAYO, M. C. S. O desafio do conhecimento: Pesquisa qualitativa em saúde. 11. Ed. São Paulo, SP: HUCITEC, 2008.

14. NOGUEIRA RS, FERNANDES VLC. O espaço saúde e lazer: a academia ao ar livre em questão. Universidade do Estado do Pará, 2013:1-15.

15. OLIVEIRA DM. Política de esporte e lazer: estudo sobre práticas corporais em participantes da Academia ao Ar Livre do Complexo Esportivo Guarani Atlântico em Santa Maria - RS, 2015: 1-11.

16. OLIVEIRA NM, SILVA DAS, TOSCANO JJO. Programas de avaliação em academias de ginástica: o que se faz?. Revista Brasileira de Atividade Física e Saúde, 2014; 19(5): 568-578.

17. OLIVEIRA RTQ, IGNÁCIO CF, NETO AHAM, BARATA MML. Matriz de avaliação de programas de promoção da saúde em territórios de vulnerabilidade social. Ciência e Saúde Coletiva, 2017; 22(12): 3915-3932.

18. ORGANIZAÇÃO MUNDIAL DE SAÚDE. United Nations Children's Fund. Cuidados Primários de Saúde Alma- Ata. URSS: OMS, 1978.

19. SALDANHA F, ZAMBIASI F, KRUG M R. Políticas públicas de promoção da saúde e qualidade de vida através da atividade física: um estudo de caso no município de Palmeira das Missões, RS. Revista Gestão Universitária, 2016; 5(1): 1-13.

20. SCHEFFER MLC, PILATTI LA, KOVALESKI JL. Qualidade Vida e Atividade Física na Literatura. Espacios, $2015 ; 36$ (3): 1-7.

21. SILVA AGF, MOTA LA, DORNELAS CSM, LACERDA AV. A relação entre Estado e políticas públicas: uma análise teórica sobre o caso brasileiro. Debates, 2017; 11 (1): 25-42

22. SILVA RN, GUARDA FRB, HALLAL PC. Avaliabilidade do Programa Academia da Saúde no município do Recife, Pernambuco, Brasil. Cadernos de Saúde Pública, 2017; 33 (4):1-16.

23. TRIVIÑOS, A.N.S. Introdução à pesquisa em ciências sociais: a pesquisa qualitativa em educação. São Paulo: Atlas, 1994, $175 p$.

24. WEBER, VMR, TEIXEIRA WM, MALFATTI CRM, SILVA LA. Avaliação da qualidade de vida de participantes do projeto "Ginástica para todos no município de Guarapuava - PR. Revista do Departamento de Educação Física e Saúde e do Mestrado em Promoção da Saúde da Universidade de Santa Cruz do Sul / Unisc, 2017; 18 (1): 45-59. 